\title{
Schistosomal myeloradiculopathy-an underdiagnosed disease: Case Report
}

\begin{abstract}
The aim of this study is to relate the condition of paraplegia due to schistosomal myeloradiculopathy with an initial diagnosis of polyradiculoneuropathy in a patient from an endemic area. This disease is the most severe and disabling ectopic form of Schistosoma mansoni infection, being underreported, of unknown prevalence and underestimated morbidity. Its recognition and the early institution of treatment play a fundamental role in the prevention of severe and irreversible injuries, as well as in the recovery of affected people, usually young people in full production phase. The diagnosis of schistosomal myeloradiculopathy is based on epidemiology, clinical findings regarding neurological conditions and evidence of Schistosome mansoni in laboratory tests such as serological techniques or stool parasitological examination and other causes for the condition should be excluded. In the case described, the patient reported swimming in a lagoon in Sergipe, an endemic zone, which makes the epidemiology speak strongly in favor of the diagnostic hypothesis. The clinical presentation presented by the patient is very similar to that of this myeloradiculopathy and that Schistosoma eggs were found in the feces, and the serology and cerebrospinal fluid analysis (CSF) serology were positive for this parasite. It is noteworthy that magnetic resonance imaging is a very sensitive propaedeutic method for the evaluation of schistosomal myeloradiculopathy, although the diagnosed alterations are not specific for this disease. The changes observed on magnetic resonance imaging (MRI) regress in parallel with the clinical improvement of the patient after treatment with schistosomicides and corticosteroids, as can be observed in this case. Finally, the treatment was done with praziquantel, ivermectin and corticosteroid therapy in association with motor physiotherapy. The patient presented clinical improvement, but maintained mild motor deficit.
\end{abstract}

Keywords: schistosome mansoni, magnetic resonance imaging, neuroschistosomiasis, paraplegia, polyradiculoneuropathy
Volume 7 Issue I - 2020

\author{
Daniela Mello Tonolli, Danielle Navarro Sato, \\ Maria Fernanda Melega Mingossi, Leticia \\ Lambert, Lorraine Lorene Felix Cardoso \\ Medicine, Centro Universitário Lusíada/Guilherme Alvaro \\ Hospital, Brazil
}

Correspondence: Daniela Mello Tonolli, Avenida Conselheiro Nébias número 663 Bairro Boqueirão, Santos, São Paulo, Brazil, Tel +55 (14)98I83-1 I09, Email dmtonoll@gmail.com

Received: January 0I, 2020 | Published: January 23, 2020
Abbreviations: WHO, world health organization; CNS, central nervous system; CREI, Centro de Referência em Emergência em Internação of São Vicente; CSF, cerebrospinal fluid analysis, MRI, magnetic resonance imaging; BALC, patient's name

\section{Introduction}

Schistosomiasis is a tropical endemic parasitic disease caused by a Schistosoma trematode flatworm, whose incubation period may vary from weeks to years. In Brazil, it is caused by Schistosoma mansoni.

In 2011, world health organization (WHO) estimated that 200 million people are infected worldwide with Schistosoma, with 65 million living in endemic areas. It is a widely distributed disease in Africa, South America, the Caribbean and the Eastern Mediterranean and is endemic in Brazil, with 1.5 million people living in our country at risk of contagion. Currently, the disease is detected in all regions of the country. The endemic and focal areas include 19 Federated Units and comprise the states of Alagoas, Bahia, Pernambuco, Rio Grande do Norte (coastal strip), Paraíba, Sergipe, Espírito Santo and Minas Gerais (predominantly in the north and northeast of the state). In Pará, Maranhão, Piauí, Ceará, Rio de Janeiro, São Paulo, Santa Catarina, Paraná, Rio Grande do Sul, Goiás and the Federal District, the transmission is focal, not reaching large areas. The CNS can be affected in infections by Schistosoma mansoni, but also by $S$ haematobium and $S$ japonicum. Asymptomatic CNS egg deposition is frequent. When symptomatic, the first two species cited most often cause myeloradicular syndrome, and the latter causes brain involvement. Schistosomal myelaryadiculopathy, the most common form of CNS disease, is most often related to schistosomiasis mansoni and is the most severe and disabling ectopic form of infection with this parasite.

\section{Case report}

BALC, 18 years old, previously healthy, from Sergipe, on December 26, 2016, started presenting fever, colic abdominal pain, vomiting, diarrhea (1 episode was bloody) and low back pain. She reported that she swam in the lagoon of her city in November 2016. She came to São Vicente-estate of São Paulo (since her mother lives in this city) and was admitted to the Centro de Referência em Emergência em Internação of São Vicente (CREI). The patient evolved with lower limbs' paresthesia and plegia, areflexia, urinary retention requiring delayed bladder catheter and fecal retention on January 23, 2017. She was then diagnosed with Guillain-Barré Syndrome, as she underwent cerebrospinal fluid analysis of January 25, 2017, proteocytological dissociation (proteins 101.4/leukocytes 30 with $90 \%$ lymphocytes and $10 \%$ neutrophils/glucose 40 ) and negative bacteriology was detected. Thus, she was treated with immunoglobulin for six days.

The patient was transferred to Guilherme Álvaro Hospital on January 28, 2017, where investigation and treatment with corticosteroid therapy (methylprednisolone for five days) was 
continued. Thoracic-lumbar spine magnetic resonance imaging (MRI) performed on January 30, 2017, was performed, which showed expansion of the spinal cord, signal alteration and anomalous contrast enhancement in the thoracic medulla, spinal cord and equine tail roots that could be related to the demyelinating disease. CSF analysis was repeated on January 31, 2017, maintaining the same result. The CSF sample was also used for protein electrophoresis, showing an increase in total proteins, with the predominance of gamma-type proteins. Stool parasitological examination of February 1, 2017, was positive for Schistosoma mansoni. Serum serology for schistosomiasis was positive, and the remaining serology was negative (hepatitis C, hepatitis B, Human Immunodeficiency Virus, syphilis, Human T-lymphotropic Virus, cytomegalovirus, toxoplasmosis), these were requested to exclude viral and bacterial myelitis. Then the diagnostic hypothesis of Schistosomiasis Myeloradiculopathy was made, and serology for CSF schistosomiasis was requested, which was positive. Then, praziquantel, ivermectin and prednisone were initiated.

Cranial magnetic resonance imaging of February 10, 2017, showed no change, while an abdominal computed tomography scan of February 6, 2017, showed enlarged dimensions and irregularly contoured liver with no intra or extrahepatic bile duct dilatation and the remaining without changes. Control thoracolumbar magnetic resonance imaging performed on February 17, 2017, demonstrated signal change in the spinal cord from T12 to the medullary cone, characterized by T1 hyposignal and T2 and STIR hypersignal, with contrast enhancing nodular foci, determining mild local expansive effect. In relation to the previous exam, a significant reduction of the lesion was noted. The set of findings was suggestive of an infectious process, among them schistosomiasis, and the demyelinating etiology cannot be ruled out.

New stool parasitological examination harvested on February 16, 2017, after treatment, continued to demonstrate Schistosoma, which, according to the assessment of Infectology, can occur even after treatment. During hospitalization, the patient developed urinary tract infections due to delayed bladder catheterization, then using antibiotic therapy (ciprofloxacin, tazocin, fluconazole). The patient presented neurological improvement during hospitalization after treatment and with motor physical therapy, being discharged in good clinical condition. She is currently in outpatient follow-up with the Neurology team at Guilherme Álvaro Hospital (Figure 1\&2).

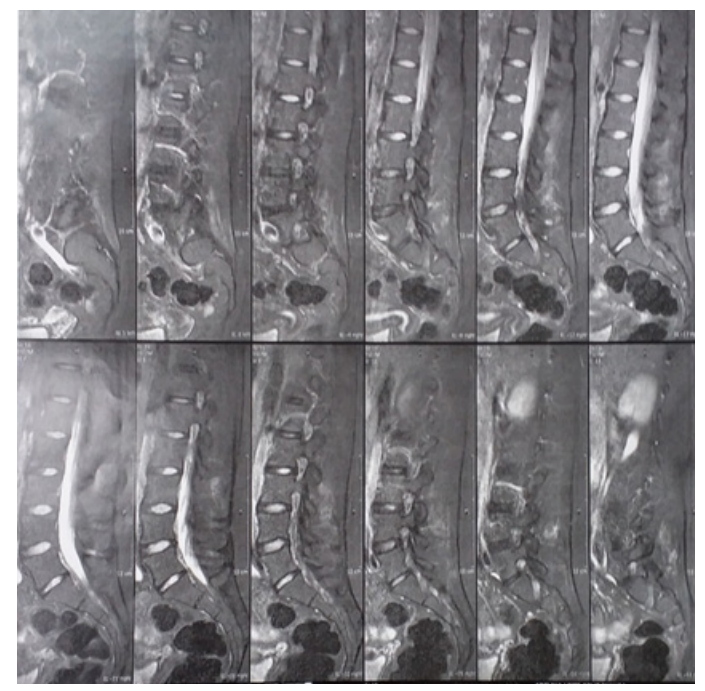

Figure I Thoracolumbar magnetic resonance imaging on 0I/30/I7.

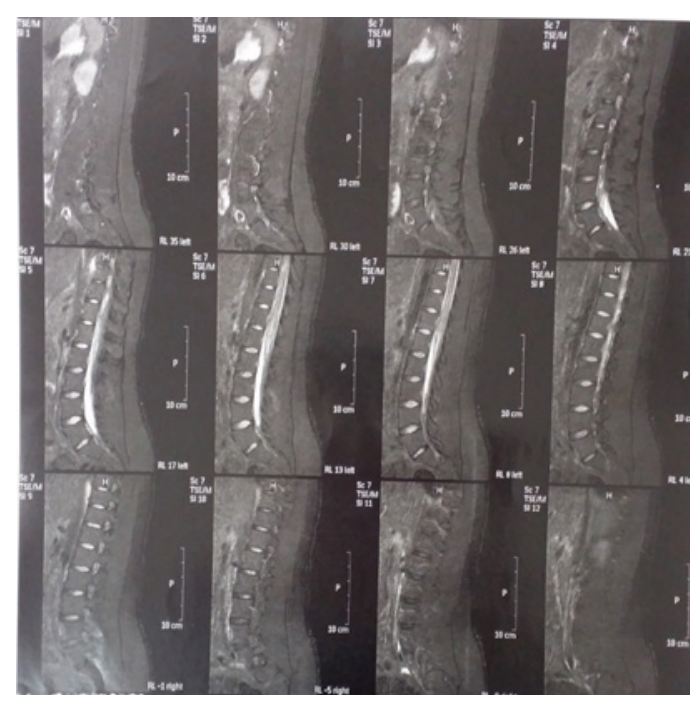

Figure 2 Thoracolumbar magnetic resonance $02 / 17 / 17$.

\section{Discussion}

The pathogenesis of schistosomal myeloradiculopathy remains unknown, but it is assumed that the inflammatory response of the host to eggs in the nervous tissue is the primary determinant of CNS lesions. ${ }^{1-3}$ The inflammatory response may range from intense host reaction, resulting in granulomas or expansive masses, to minimal reaction without clinical expression. In some necropsied cases, eggs are found in the medullary tissue without any inflammatory response. The contribution of an autoimmune process triggered by schistosomal infection, causing vasculitis and ischemia, should also be admitted, but there is a lack of data to confirm such hypothesis. Asymptomatic deposition of $S$ mansoni eggs in both the brain and spinal cord was more common than the symptomatic form of the disease.

Myeloradiculopathy occurs most often due to the acute or chronic intestinal form of worms; however, there are reports in people with hepatosplenic form. ${ }^{3}$ Eggs and worms may travel through the avalvular Batson epidural vertebral venous plexus, which connects the portal system and the vena cava to the veins of the spinal canal. Thus, the eggs reach the CNS through local oviposition or embolization. The higher incidence of myelopathy in the lumbosacral region is probably explained by this migration mechanism. Besides, autopsy studies have shown that $S$ hematobium eggs are more frequently deposited in the brain than $S$ mansoni eggs. Eggs of this latter species are larger than $S$ hematobium eggs $(155 \times 66 \mu \mathrm{m}$ compared to $143 \times 55 \mu \mathrm{m})$ and it has been suggested that the lateral spicule of $S$ mansoni egg may also impede its progression through blood vessels. These are hypotheses put forward to justify the tendency for $S$ mansoni eggs to lodge in the lower regions of the vertebral venous plexus.

The clinical picture of the patient corroborates these data since she presented the intestinal form of schistosomiasis disease evolving to CNS involvement and in addition, she presented the spinal cord form, which is the most prevalent, but also the most frequently symptomatic, whose symptoms general include fever, headache, myalgia, diarrhea and abdominal pain; being the classic prodromal clinical triad: low back pain, change in strength and/or sensibility of the lower limbs and urinary disorder. In our report, the case began with fever, abdominal pain, diarrhea, vomiting and low back pain that evolved to flaccid lower limb paresthesia and plegia, areflexia, and urinary and fecal retention. 
Recalling that paraplegia with flaccidity and areflexia, urinary retention and reduced tactile, thermal and painful sensitivity of the root type dominate the clinical picture when the medullary cone and the equine tail are affected, as we saw in the reported case. Spasticity, alteration of the superficial sensitivity of the segmental level type and urinary incontinence occur in the presence of higher involvement of the spinal cord. In general, bilateral paraparesis and deep reflexes abolished or reduced are the most frequent findings on neurological examination.

It is noteworthy that the diagnosis of schistosomal myeloradiculopathy is based on epidemiology, clinical findings regarding neurological conditions and evidence of Schistosome mansoni in laboratory tests such as serological techniques or stool parasitological examination and other causes for the condition should be excluded. In the case described, the patient reports a history of swimming in a lagoon in Sergipe, an endemic zone, which makes the epidemiology speak strongly in favor of the diagnostic hypothesis. The other two pillars of the diagnosis of the disease also speak in support of this case: since the clinical presentation presented by the patient is very similar to that of this myeloradiculopathy and that Schistosoma eggs were found in the feces and the serology and CSF serology were positive for this parasite.

CSF analysis of patients with schistosomal myeloradiculopathy reveals unspecific changes: slight to moderate elevation of protein content in $95 \%$ of cases (mean $161.4 \pm 191.9 \mathrm{mg} / \mathrm{dl}$ ), normal glucose levels, pleocytosis in $91 \%$ of the cases (average of $91.9 \pm 113.8$ cells/ $\mathrm{mm}^{3}$ ), with predominance of lymphomononuclear cells, presence of eosinophils in 41 to $90 \%$ of cases and elevation of gamma globulin in $76 \%$ of cases. ${ }^{2}$ Andrade Filho et al. observed a marked reduction in liquor cellularity with an improvement of symptoms, but persistent hyperproteinorrhage in $66 \%$ of cases. Anti-Schistosoma antibodies were identified in 85 to $90 \%$ of CSFs tested using ELISA, indirect immunofluorescence or hemagglutination techniques. The positivity of CSF Schistosoma serology is considered evidence of schistosomal CNS infection by several authors. Livramento, Machado e Silva described the cerebrospinal fluid syndrome in neuroschistosomiasis, an important element in the presumptive clinical and laboratory diagnosis of schistosomiasis myeloradiculopathy: lymphomononuclear pleocytosis, hyperproteinorrachia, and positivity of specific immunological reactions for schistosomiasis (immunofluorescence, ELISA or haemagglutination). The presence of CSF antibodies to $S$ mansoni-associated with cellularity $>4$ cells $/ \mathrm{mm},{ }^{3}$ protein rate $>40 \mathrm{mg} /$ $\mathrm{dl}$ and the presence of eosinophils-suggests a high probability of this disease. The patient presented these changes in CSF analysis (except for the increase in eosinophils, as they were not quantified) and also presented positive CSF serology. ${ }^{4-9}$

The search for antibodies against serum Schistosoma antigens for the diagnosis of infection has limitations due to the cross-reaction with other antigens, especially other helminthes and the difficulty of differentiating it from active infection from previous contact with the parasite, because the antibodies may remain in the serum for long periods even after curative treatment. ${ }^{10}$ Nevertheless it was done and was positive. The main alterations described in magnetic resonance imaging in this pathology are: increased spinal cord and/or equine tail diameter in T1-weighted images; T2 signal hyperintensity in the affected region, representing increased water content (edema); and heterogeneous contrast uptake (possibly homogeneous) due to the breakdown of the blood-brain barrier, with small focal areas of accentuation often forming a granular pattern. Therefore, it is a very sensitive propaedeutic method for the evaluation of schistosomal myeloradiculopathy, although the diagnosed alterations are not specific for this disease. The changes observed on MRI regress in parallel with the clinical improvement of the patient after treatment with schistosomicides and corticosteroids, as can be observed in this case.

Importantly, the occurrence of schistosomal myeloradiculopathy, unlike other severe forms of schistosomiasis, such as hepatosplenic and pulmonary hypertension, does not depend on high parasitic loads. In contrast, patients with this disease usually have few eggs per gram of feces and often come from low prevalence areas. Finally, the treatment of schistosomal myeloradiculopathy is with schistosomicides (ivermectin and praziquantel), corticosteroids and/or surgery. The association of schistosomicide with corticosteroids is related to the best therapeutic response in the disease. ${ }^{3,4,6,7,11}$ There would be both an action against the etiologic agent and an anti-inflammatory action by the corticoid, which may contribute to the improvement of the neurological condition. The prognosis depends on the rapid use of these drugs, so early diagnosis is of utmost importance. About $95 \%$ of patients with an untreated schistosomal myeloradiculopathy die or show no clinical improvement. In one study, ${ }^{4}$ instituting therapy, even late, after 12 months of the neurological picture, was associated with neurological improvement. In this case, treatment was started early and associated with motor physiotherapy there was a satisfactory evolution of the disease.

\section{Conclusion}

This disease is the most severe and disabling ectopic form of Schistosoma mansoni infection, being underreported, of unknown prevalence and underestimated morbidity. Its recognition and the early institution of treatment play a fundamental role in the prevention of severe and irreversible injuries, as well as in the recovery of affected people, usually young people in full production phase.

\section{Acknowledgments}

None.

\section{Funding}

None.

\section{Conflicts of interest}

Author declares that there is no conflict of interest.

\section{References}

1. Ministry of Health. Guide to epidemiological surveillance and control of schistosomal myelorradiculopathy. Brazil; 2006.

2. Livramento JA, Machado LR, Silva CL. Cerebrospinal fluid syndrome in neuroschistosomiasis. Arq Neuro-Psychiatrist. 1985;43:372-377.

3. Silva LCS. Schistosomal myelorradiculopathy. Rev Soc Bras Med Trop. 2004;39(3):261-272.

4. Silva LC, Maciel PE, Ribas JG, et al. Treatment of schistosomal myeloradiculopathy with praziquantel and corticosteroids and evaluation by magnetic resonance imaging: a longitudinal study. Clin Infect Dis. 2004;39(11):1618-1624. 
5. Lambertucci JR, Silva LC, do Amaral RS. Guidelines for the diagnosis and treatment of schistosomal myeloradiculopathy. Rev Soc Bras Med Trop. 2007;40(5):574-581.

6. Lambertucci JR, Serufo JC, Gerspacher-Lara R, et al. Schistosoma mansoni: assessment of morbidity before and after control. Acta Tropica. 2000;77(1):101-109.

7. Peregrino AJP. Meningomyeloradiculitis by Schistosoma mansoni: investigation protocol and report of 21 cases. Arq Neuro-Psychiatrist. $1988 ; 46: 4960$

8. JR Coura, RS Amara. Epidemiological and control aspects of schistosomiasis in Brazilian endemic areas. Mem Inst Oswaldo Cruz. 2004;99(supl 1):13-19.
9. Pammenter MD, Epstein SR, Rees RT. Cross reactions in the immunodiagnosis of schistosomiasis and cysticercosis by a cerebrospinal fluid enzyme-linked immunosorbent assay. Trans $R$ Soc Trop Med Hyg. 1992;86:51-52.

10. Lambertucci JR, Sousa-Pereira SR, Silva LC. Myeloradiculopathy in acute schistosomiasis mansoni. Rev Soc Bras Med Trop. 2005;38(3):277-278.

11. Peregrino AJP. Medular Schistosomosis Analysis of 80 cases. Arq Neuro Psychiatrist. 2002;60(3):603-608. 